\title{
Posters also presented at the Symposium
}

\author{
3D Hydrodynamic Simulations of O-Shell Convection \\ Robert Andrassy \\ Department of Physics and Astronomy, University of Victoria
}

I am reporting on our team's progress in investigating fundamental properties of convective shells in the deep stellar interior during advanced stages of stellar evolution. We have performed a series of 3D hydrodynamic simulations of convection in conditions similar to those in the O-shell burning phase of massive stars. We focus on characterizing the convective boundary and the mixing of material across this boundary. Results from $768^{3}$ and $1536^{3}$ grids are encouragingly similar (typically within 20\%). Several global quantities, including the rate of mass entrainment at the convective boundary and the driving luminosity, are related by scaling laws. We investigate the effect of several of our assumptions, including the treatment of the nuclear burning driving the convection or that of neutrino cooling. The burning of the entrained material from above the convection zone could have important implications for pre-supernova nucleosynthesis.

\section{The Upper Initial Mass Function in Nearby Dwarf Galaxies J. Andrews, D. Calzetti, D. Cook, D. Dale, and M. Krumholz University of Arizona}

Dwarf galaxies are likely the building blocks of all galaxies observed today, but star formation is still poorly-understood in these unevolved systems. We have obtained groundbased optical spectroscopy with the MMT and Palomar-200 inch for a sample of young, $\mathrm{H}$-alpha bright clusters in $\sim 20$ dwarfs within $\sim 3.5 \mathrm{Mpc}$; our targets all have extensive HST broad-band coverage as well. By comparing these extinction corrected spectra to stochastic stellar synthesis models we can obtain ages and masses of the clusters and associations and determine whether the dearth of ionizing photons in dwarfs is an effect of deficiency in the production of massive stars. Here we will present the results of those observations, and show that stellar clusters with massive stars are not as rare as generally thought, and are commonly found in smaller clusters in the low metallicity dwarfs.

\section{Constraining Disk Properties of a Survey of Southern Be Stars Arcos, C. ; Kanaan, S. ; Curé, M.; Universidad de Valparaíso}

Be stars represent a challenge in the study of the physical mechanism required to form and support disks around hot stars. Using different techniques, such as photometry, interferometry and spectroscopy and thanks to the development of powerful radiative codes, we can study the conditions for which disk exist as well as disk features such as the size of the emission region, inclination angle, mass, angular momentum, etc. Using BEDISK a non-LTE radiative transfer code and BERAY that solves the transfer equation throughout the disk, we studied 63 Be southern stars by modeling $H_{\alpha}$ emission line 
profiles. We found for early stellar emission types that the density structure in the surface of the disk can be modeled by a power law with base density between $\rho_{0} \sim(4.00$ - 6.30) $\times 10^{-11} \mathrm{~g} \mathrm{~cm}^{-3}$ with a power-law fall of between $n \sim 2.0-2.5$. We also found that outer disk radii of the $H_{\alpha}$ emitting regions are between $R_{d i s k} \sim 20-30 R_{\star}$, the angular momentum between $J_{d} \sim(1.0-3.1) \times 10^{-7} J_{\star}$ and the disk mass between $M_{d} \sim(1.0$ $3.1) \times 10^{-9} M_{\star}$.

\section{RADFLAH: A New, Open Numerical Framework to Model Supernova Light Curves with the FLASH Code Chatzopoulos, E., Wheeler, J. C., Vinko, J. Louisiana State University}

We present the newly-incorporated gray radiation hydrodynamics capabilities of the FLASH code based on a radiation flux-limiter aware hydrodynamics numerical implementation designed specifically for applications in astrophysical problems. The newly incorporated numerical methods consist of changes in the pristine unsplit hydrodynamics solver using operator splitting and adjustments in the flux-limited radiation diffusion unit. Our method can treat problems in both the strong and weak radiation-matter coupling limits as well as transitions between the two regimes. Appropriate extensions in the "Helmholtz" equation of state are implemented to treat two-temperature astrophysical plasmas involving the interaction between radiation and matter. A set of radiationhydrodynamics test problems is presented aiming to showcase the new capabilities of FLASH and to provide direct comparison to similar codes like CASTRO. To illustrate the capacity of FLASH to simulate phenomena occurring in stellar explosions, such as shock break-out, radiative precursors and ejecta heating due to the decays of radioactive nickel and cobalt, we also present an 1D supernova simulation yielding a model gray light curve. The latest public release of FLASH with these enhanced capabilities is freely available for download and use by the broader astrophysics community.

\section{The X-Ray Origin of the Be Star Gamma Cassiopeiae and the Implication to Its Stellar Evolution \\ Michael F. Corcoran, Neetika Sharma, Ted Gull, Hiromitsu Takahashi, Christopher M. Russell, Tom Madura, Anthony Moffat, Takayuki Yuasa, Julian Pittard, Manabu ISHIDA, Jose Groh, Stan Owocki, Noel Richardson CRESST NASA/GSFC \& UMBC}

Gamma Cassiopeiae is an enigmatic Be star with unusually hard, strong X-ray emission compared with normal main-sequence B stars. The origin is controversial for decades between two theories: mass accretion onto a hidden compact companion and magnetic dynamo driven by the star-Be disk differential rotation. There has been found no decisive signature that support either theory, such as a pulse in X-ray emission or the presence of large-scale magnetic field. In a $\sim 100 \mathrm{ksec}$ duration observation of the star with the Suzaku X-ray observatory in 2011, we detected six rapid X-ray spectral hardening events called "softness dips". All the softness dip events show symmetric softness ratio variations, and some of them have flat bottoms apparently due to saturation. The softness dip spectra are best described by either $\sim 40 \%$ or $\sim 70 \%$ partial covering absorption to $\mathrm{kT} \sim 12 \mathrm{keV}$ plasma emission by matter with a neutral hydrogen column density of 
$\sim 2-8 \times 10^{21} \mathrm{~cm}^{-2}$, while the spectrum outside of these dips is almost free of absorption. This result suggests that two distinct X-ray emitting spots in the gamma Cas system, perhaps on a white dwarf companion with dipole mass accretion, are occulted by blobs in the Be stellar wind, the Be disk, or rotating around the white dwarf companion. The formation of a Be star and white dwarf binary system requires mass transfer between two stars; gamma Cas may have experienced such activity in the past. We discuss the gamma Cas type Be stellar evolution, based on this result.

\section{Connecting Nuclear Physics and Massive Star Models to Galactic Chemical Evolution \\ Benoit Cote, Christian Ritter, Falk Herwig, Brian W. O'Shea, Chris L. Fryer \\ University of Victoria / Michigan State University}

Modeling the chemical evolution of the Milky Way and Local galaxies represents a significant challenge, as it contains several sources of uncertainties and ideally requires multidisciplinary collaborations. Nuclear physics provides nuclear reaction rates, stellar models provide the composition of stellar ejecta, galaxy models and simulations follow the evolution of chemical species driven by multiple stellar populations, and observations provide constraints to test and improve numerical recipes. Continuous communication and feedback between all these fields is a key component in improving our understanding of how, where, and when elements have been created and recycled across cosmic time. We built a numerical pipeline that connects NuGrid stellar yields to galactic chemical evolution models, going from a classical one-zone model to a multi-zone model able to post-process cosmological hydrodynamical and dark-matter-only simulations. During this talk, I will present how we used this pipeline, in a circular way, to probe the impact of nuclear astrophysics and massive star evolution assumptions on the chemical signatures predicted by chemical evolution models. I will highlight the importance of core-collapse nucleosynthesis and how the uncertainties associated with the stellar remnant mass and black hole formation prescriptions in massive star models affect our ability to provide reliable numerical predictions in the Milky Way and in dwarf spheroidal galaxies.

\section{D MHD Simulations of Radiatively Driven Winds with Inclined Magnetic Fields \\ Simon Daley-Yates \\ Astrophysics and Space Research Group, University of Birmingham}

We present results of $2 \mathrm{D}$ and $3 \mathrm{D}$ numerical simulations of magnetically confined, radiatively driven stellar winds of massive stars, conducted using the astrophysical MHD code Pluto, with a focus on understanding the rotational variability of radio and sub-mm emission. Radiative driving is implemented according to the Castor, Abbott and Klein theory of radiatively driven winds. Many magnetic massive stars posses a magnetic axis which is inclined with respect to the rotational axis. This misalignment leads to a complex wind structure as magnetic confinement, centrifugal acceleration and radiative driving act to channel the circumstellar plasma into a warped disk whose properties should be apparent in multiple wavelengths. Building upon work carried out by ud-Doula, Owocki and Townsend, simulating magnetically channeled radiatively outflow. Light curves in 
multiple wavelengths via Monte Carlo radiative transfer are presented. Parameters such as the mass-loss rate, magnetic field strength, rotational period, magnetic and rotational axis misalignment are investigated.

\section{Photometric Variability of Luminous Blue Variables in M33 on Short Timescales \\ Gantcho Gantchev, Petko Nedialkov, Valentin Ivanov, Evgeni Ovcharov, Antoniya Valcheva, Milen Minev \\ Department of Astronomy, Physics Faculty, Sofia}

We used SDSS r-band aperture photometry and astrometry of 500 000 stellar-like objects in the M33 galaxy performed by the CASU (Cambridge Astronomy Survey Unit) Astronomical Data Centre in the Institute of Astronomy, University of Cambridge. The observations were carried out with the $2.6 \mathrm{~m}$ VISTA telescope at the Cerro Paranal, Chile. More than 500 images in that passband were obtained with the OmegaCAM, a large format $(16 \mathrm{k} \times 16 \mathrm{k}$ pixels $) \mathrm{CCD}$ camera, and each of them covers a field of view of $1^{\circ} \times 1^{\circ}$. The current time span of the data is $2.1 \mathrm{yrs}$ until the end of 2014 . The structure function analysis (Hughes et al. 1992) was applied in order to study the variability of $\sim 30$ known or suspected LBVs in the M33 galaxy (Massey et al. 2007) on different time scales. In some cases like Var $\mathrm{C}$ the time resolution of the data allows us to confirm an enhanced weekly variations $\mathrm{Dm} \sim 0.3 \mathrm{~m}$ which is somehow shorter than the previously know typical monthly variations with the same maximum amplitude thought to be caused by non-radial pulsations.

\section{Diversified Core-Collapse Supernovae Through A Jet-Feedback Mechanism Avishai Gilkis, Noam Soker \\ Physics Department, Technion - Israel Institute of Technology}

I propose that the energy output of explosions following massive stellar core-collapse is regulated through a jet-feedback mechanism. This scenario advocates for jet-powered central engines producing different outcomes depending on the efficiency of the interaction between the jets and their surrounding. An efficient coupling of the jets with the infalling material will result in the halt of material accretion and the central engine shutting down early-on, resulting in a regular energy supernova. A lower efficiency will allow a longer accretion period and a higher energy output from the central engine, possibly resulting in black hole formation and superluminous supernovae or gamma-ray bursts. I demonstrate these concepts on models of massive stars with the aid of analytic approximations and numerical codes, and discuss potential consequences of this model.

\section{Wolf-Rayet Stars in M81: Detection and Characterization Using GTC/OSIRIS Spectra and HST/ACS Images \\ Víctor Mauricio Alfonso Gómez-Gonzólez, Yalia Divakara Mayya, Daniel Rosa Gonzólez \\ Instituto Nacional de Astrofísica, Óptica y Electrónica}

We here report the properties of Wolf-Rayet (W-R) stars in 14 locations in the nearby spiral galaxy M81. These locations were found serendipitously while analysing the slit 
spectra of a sample of $\sim 150$ star-forming complexes, taken using the long-slit and MultiObject spectroscopic modes of the OSIRIS instrument at the $10.4 \mathrm{~m}$ Gran Telescopio Canarias. Colours and magnitudes of the identified point sources in the Hubble Space Telescope images compare well with those of individual W-R stars in the Milky Way. Using templates of individual W-R stars, we infer that the objects responsible for the observed W-R features are single stars in 12 locations, comprising of 3 WNLs, 3 WNEs, 2 WCEs and 4 transitional WN/C types. In diagrams involving bump luminosities and the width of the bumps, the W-R stars of the same sub-class group together, with the transitional stars occupying locations intermediate between the WNE and WCE groups, as expected from the evolutionary models. However, the observed number of 4 transitional stars out of our sample of 14 is statistically high as compared to the $4 \%$ expected in stellar evolutionary models.

\section{The Yellow and Red Supergiants of M31/M33 and Post-Red Supergiant Evolution}

\section{Michael S. Gordon, Roberta Humphreys, and Terry J. Jones Minnesota Institute for Astrophysics}

From surveys of luminous star populations in nearby galaxies, we have selected a sample of yellow and red supergiant candidates in M31 and M33 for study of their spectral characteristics and spectral energy distributions to place them on an HR Diagram. As the position of intermediate and late-type supergiants on the color-magnitude diagram can be heavily contaminated by foreground dwarfs, we used spectral classification and multi-band photometry from optical and near-infrared surveys to confirm membership, in addition to careful measurements of the extinction along the line-of-sight to each source to determine bolometric luminosities. Based on spectroscopic evidence for stellar winds and mass loss and the presence of circumstellar dust in their SEDs, we find that 30-40\% of the yellow supergiants in M31 and M33 are likely in a post-red supergiant state. Comparison with evolutionary tracks suggests that these mass-losing, post-RSGs have initial masses between $20-40 M_{\odot}$. More than half of the observed red supergiants are producing dusty circumstellar ejecta, and thus may be more likely to evolve back to warmer temperatures due to their high mass loss.

\section{Feedback by Massive Stars and the Self-Regulation of Star Formation Gerhard Hensler \\ Dept. of Astrophysics, Univ. of Vienna}

Although star formation (SF) in action becomes directly noticeable in galaxies by various observational signatures, its process, its empirical relations to gas properties, and the reason for a much lower SF rate than expected are still far from being understood in detail. Most importantly, massive stars act as the main driver of galaxy evolution by various modes of stellar feedback, energy, mass and element releases, the first regulating the SF and energizing the interstellar medium (ISM), the last one enriching the ISM with heavier elements. For cosmological and galaxy evolutionary models the "star-formation efficiency" is an essential parameter to describe which fraction of star-forming gas is really converted into stars. Instead as being assumed temporally and empirically constant, this parameter depends not only on the stellar energy release, but also on the conditions of the formed star cluster and its surrounding gas. The formation of extremes like super 
star clusters as those in starburst galaxies and required previously for Globular Clusters as well as SF in low-surface brightness galaxies prove this conclusion. From our former numerical models of radiation-driven and wind-blown HII regions around single massive stars (Freyer et al.) we evaluate the energy transfer efficiency and found much lower values than those generally assumed in galaxy evolution modeling. Moreover, ongoing numerical studies of lacking massive stars at very low SF rates has also strong consequences for our understanding of feedback and SF self-regulation. Both will be discussed in our presentation.

\section{Stellar parameters of the reference O-type stars Anthony Hervé Stellar Department ASU}

Massive stars play a key role in various fields of astrophysics. In the 70's, the two dimensional spectral classification of O stars was developed by Walborn. Standard stars have been selected to be the reference object of each stellar type / luminosity class. These standard stars are still used today for the classificationof the any newly discovered O stars. However, the stellar properties of these reference objects have never been determined in a homogeneous way. Using high-resolution, high signal-to-noise ratio from OHP and ESO and the modeling code CMFGEN, we are currently determining the properties of the standard O-type stars. During this presentation we will present the preliminary results of our study.

\section{Rising from the Ashes: Mid-Infrared Re-Brightening of the Impostor SN 2010da in NGC 300}

\section{Ryan M. Lau, Mansi M. Kasliwal, Howard E. Bond, et al.} Caltech/JPL

We present multi-epoch mid-infrared (IR) photometry and the optical discovery observations of the "impostor" supernova (SN) 2010da in NGC 300 using new and archival Spitzer Space Telescope images and ground-based observatories. The mid-IR counterpart of SN 2010da was detected as SPIRITS 14bme in the SPitzer InfraRed Intensive Transient Survey (SPIRITS), an ongoing systematic search for IR transients. A sharp increase in the $3.6 \mu \mathrm{m}$ flux followed by a rapid decrease measured $\sim 150 \mathrm{~d}$ before and $\sim 80$ $\mathrm{d}$ after the initial outburst, respectively, reveal a mid-IR counterpart to the coincident optical and high luminosity X-ray outbursts. At late times after the outburst $(\sim 2000$ d), the 3.6 and $4.5 \mu \mathrm{m}$ emission increased to over a factor of 2 times the progenitor flux. We attribute the re-brightening mid-IR emission to continued dust production and increasing luminosity of the surviving system associated with SN 2010da. We analyze the evolution of the dust temperature, mass, luminosity, and equilibrium temperature radius in order to resolve the nature of SN 2010da. We address the leading interpretation of SN 2010da as an eruption from a luminous blue variable (LBV) high-mass X-ray binary (HMXB) system. We propose that SN 2010da is instead a supergiant (sg)B[e]-HMXB based on similar luminosities and dust masses exhibited by two other known sgB[e]HMXB systems. Additionally, the SN 2010da progenitor occupies a similar region on a mid-IR color-magnitude diagram (CMD) with known sgB[e] stars in the Large Magellanic Cloud. The lower limit estimated for the orbital eccentricity of the sgB[e]-HMXB $(e>0.82)$ from X-ray luminosity measurements is high compared to known sgHMXBs 
and supports the claim that SN 2010da may be associated with a newly formed HMXB system.

\section{Chandra X-Ray Grating Spectroscopic Diagnostics of the X-Ray Emitting Plasma in the Magnetic O+O Binary Plaskett's Star}

M. Leutenegger, J. Grunhut, G. Wade, D. Cohen, T. Doyle, M. Gagne, R. Ignace, J. C. Leyder, L. Mahy, T. Moffat, Y. Naze, S. Owocki, V. Petit, J. Sundqvist, A. ud-Doula

\section{NASA/Goddard Space Flight Center Code 662}

Recent spectropolarimetric observations have revealed that the rapidly rotating secondary in the massive $\mathrm{O}+\mathrm{O}$ binary system Plaskett's star harbors a strong magnetic field with a surface dipole field strength of $\sim 3 \mathrm{kG}$ (Grunhut et al. 2013), suggesting the likely presence of a centrifugally supported magnetosphere, unique among $\mathrm{O}$ stars. Previous $\mathrm{X}$-ray observations interpreted the X-ray emission in terms of a collision between the primary and secondary wind (Linder et al. 2006), but the presence of a strong magnetic field on the secondary suggests that X-ray emission might originate from the magnetosphere or even in a wind magnetosphere collision. To distinguish between these competing hypotheses, we have obtained high resolution Chandra grating spectra of Plaskett's star, with good orbital and rotational phase coverage. The forbidden-to-intercombination line ratios of He-like Si XIII and Mg XI rule out formation of X-ray emitting plasma inside the magnetosphere of the secondary, and are consistent with formation in the region between the two stars. The lines are broad, with a Gaussian sigma of $\sim 700 \mathrm{~km} / \mathrm{s}$. We surprisingly find no evidence for variability of the line shape or shift with either secondary rotational phase or orbital phase. This is contrary to expectations for either a magnetospheric or a colliding wind origin for the X-rays.

\section{The Progenitor Masses of $\sim 100$ Core-Collapse SNe \\ Jeremiah Murphy, Mariangelly Díaz Rodríguez Physics, Florida State University}

By age-dating the stellar populations in the vicinity of supernova remnants (SNRs), we derive the progenitor masses for more than 200 core-collapse SNe. With this large statistical sample, we are able to characterize the distribution of progenitor masses. Using Bayesian statistical inference, we find that the minimum mass of SNR progenitors is $7.2 \pm 0.3$ solar masses, the maximum mass is $33_{-6}^{+17}$ solar masses, and the power law slope in between is $2.8 \pm 0.5$, consistent with the Saltpeter IMF. The accuracy of the minimum mass may provide interesting constraints on stellar evolution. With regard to the maximum mass, either the most massive of massive stars are not exploding, or there is severe bias against forming SNRs by the explosions of the most massive stars.

\section{Analytic Conditions for Core-Collapse Supernova Explosions Jeremiah Murphy, Joshua Dolence, and Quintin Mabanta \\ Department of Physics, Florida State University}

We derive an integral condition for core-collapse supernova explosions and use it to construct a new diagnostic of explodability. The fundamental challenge in core-collapse 
supernova theory is to explain how a stalled accretion shock revives to explode a star. In this talk, we assume that shock revivalis initiated by the delayed-neutrino mechanism and derive an integral condition for shock expansion, $v_{s}>0$. In general, there are five parameters in the core-collapse problem: the neutrino luminosity, the temperature of neutrinos, the neutron star radius, the neutron star mass, and the accretion rate onto the stalled shock. The integral condition represents an explosion condition that incorporates these five parameters, and this integral condition is represented by a single equation, which is characterized by a single dimensionless parameter, $\Psi>0 . \Psi=0$ defines a critical five-dimensional hypersurface, below this surface, stalled-shock solutions exists, and above this hypersurface only $v_{s}>0$ solutions exist. Therefore, $\psi=0$ defines a critical hypersurface forexplosion, and we show that the critical neutrino luminosity curve proposed by Burrows \& Goshy 1993 is a projection of this more generalcritical condition. Finally, we propose and verify with $1 \mathrm{D}$ simulations that $\Psi>0$ is a reliable and accurate explosion diagnostic.

\section{The Spectral Temperature of Light Echoes From Eta Carinae's Giant Eruption \\ Stan Owocki \& Nir Shaviv \\ Dept. of Physics and Astronomy, U. of Delaware}

The 1840's era giant eruption of eta Carinae remains a challenge for understanding the lives and death throes of the most massive stars. In recent years the detection by Rest et al. (2012) of light echoes from this era has provided important new observational constraints on the nature of the eruption. In particular, spectra of the echoes suggest a relative cool spectral temperature of about $5500 \mathrm{~K}$, below the lower limit of about $7000 \mathrm{~K}$ derived from the optically thick wind outflow analysis of Davidson (1987). This has lead to a debate about the viability of this steady wind model relative to alternative, explosive scenarios. Here we present an updated analysis of the wind outflow model using newer low-T opacity tabulations and accounting for the stronger mass loss associated with the $>10 M_{\odot}$ mass now inferred for the Homunculus. A major conclusion is that, because of the sharp drop in opacity due to free electron recombination for $\mathrm{T}<6500 \mathrm{~K}$, a low spectral temperature of about $5000 \mathrm{~K}$ is compatible with, and indeed expected from, a wind with the extreme mass loss inferred for the eruption. Within a spherical gray model in radiative equilibrium, we derive spectral energy distributions for various assumptions for the opacity variation of the wind, and make initial comparisons with observed light echo spectra.

\section{A Very Luminous Rosetta Stone to Decipher Massive Stellar Evolution: Linking LBVs, SN Impostors, Bright Radio Emitters and ULXs Manfred W. Pakull}

\section{Observatoire Astronomique de Strasbourg}

The subject of this talk is a previously unknown, very luminous $\left(M_{\mathrm{V}} \sim-10\right)$ evolved star embedded in a supergiant HII region in the nearby dwarf galaxy NGC 5408. H $\alpha$ has a HWZI of $\sim 5000 \mathrm{~km} / \mathrm{s}$ and the equivalent width is presently $2000 \AA$. It displays nonthermal radio emission which is 8 times more powerful than the Cas A SNR, and long-slit spectra reveal a large surrounding HeIII region (300 pc diameter) with intense HeII 4686 and $[\mathrm{Nev}] 3426$ emission (ionisation potential 54 and $97 \mathrm{eV}$, respectively). This points 
to the presence of an ultraluminous X-ray source $\left(L_{\mathrm{X}} \sim\right.$ several $10^{4} 0 \mathrm{erg} / \mathrm{s}$, hereafter referred to as "NGC 5408 X-2"), whereas Chandra only detected a 3 orders of magnitude lower flux level. Curiously, this object is very close to, but not identical with the famous ULX NGC 5408 X-1, which is currently being studied in much detail in the literature. I will present ESO/VLT medium resolution spectra of "X-2" revealing variable P Cygni profiles of $\mathrm{H}$, HeI and various ionisation stages of (forbidden) metal lines, suggesting a hot LBV nature. However, its optical continuum level appears to be constant whereas the $\mathrm{H} \alpha$ emission had increased tenfold over the last 30 years. The system appears to be in a very rapid stage of stellar evolution with currently ongoing huge mass-loss/mass-transfer between the binary components. LBV/ULX NGC 5408 X-2 appears to be a Rosetta stone worthwhile to be deciphered for a better understanding of the latest phases of massive (binary) stars.

\section{A revised magnetic topology for the magnetic O-type star $\theta^{1}$ Ori C V. Petit, P. Mohanty, G. Wade Florida Institute of TEchnology}

$\theta^{1}$ Orionis $\mathrm{C}$ was the first O-type star to be measured to host a magnetic field at its surface, through circular polarization induced by the Zeeman effect. Since then, the discovery and systematic characterization of magnetic fields in OB stars by the new generation of powerful spectropolarimeters has enabled a new understanding of the influence of magnetism on their radiation-driven stellar winds, and on their observed spectral characteristics at all wavelengths. However $\theta^{1}$ Orionis $\mathrm{C}$, the usual benchmark of magnetic O-type stars, has yet to be characterized with modern spectropolarimetric observations. We present a new ESPaDOnS monitoring of this poster-child object and a new determination of its surface field topology.

\section{The Berkeley Sample of Stripped-Envelope Supernovae: 20+ Years of Spectroscopy and Photometry Isaac Shivvers, Alexei Filippenko \\ Astronomy Department, University of Berkeley at California}

Stripped-envelope supernovae (SNe of types IIb/Ib/Ic, as well as some rarer subtypes) are associated with the core collapse of some of the most massive stars in the universe. These SNe exhibit heterogenous properties in their spectral and photometric evolution, the study of which is confounded by the scarcity of well-observed examples (these subtypes, on average, are both less luminous and less volumetrically common than SNe of types II and Ia). I will present the ongoing effort at Berkeley to analyze, understand, and make public the large sample of stripped-envelope SNe observations accumulated by the Filippenko group - data both previously published and as-yet unpublished, including $700+$ spectra of $250+$ SNe.

\section{The Impacts of Pre-Collapse Structures In CCSNe Theory Yu Yamamoto, Kotaro Fujisawa \\ Department of Physics and Applied Physics, Waseda University}

The main topic of CCSNe theory is now starting to pay attention to what is exact condition of powerful explosion and particularly what kind of pre-collapse structure expedite 
to obtain the canonical explosion, i.e. "initial problem". So far the practical stellar evolution calculations of massive stars are, however, still in progress due to the complicated convection theory, the effect of rotation, the presence of mass loss events and the resolution dependence. In Yamamoto et al. (2016), we studied the influence of the progenitor structure on the dynamics systematically by constructing progenitor models parametrically instead of employing realistic models provided by stellar evolution calculations. The mass of the iron core and that of silicon plus surfer $(\mathrm{Si}+\mathrm{S})$ layer are chosen as the parameters and dynamical evolutions after collapse are calculated in one and two dimensions. We found that the explosion energy is tightly correlated with mass accretion rate at shock revival irrespective of dimension and the lighter iron cores but with rather high entropies, which are yet to be produced by realistic stellar evolution calculations, may produce the more energetic explosions and the larger amounts of nickel masses. Our simulation confirmed necessity of early time explosion to reproduce $1051 \mathrm{erg}$. The authors further studied the parametric pre-collapse structures by altering the entropy distributions in core so as to see the difference in the time evolution of mass accretion rates. Furthermore, the models are also extended to shellular rotation stars by employing an approach in Fujisawa et al. (2015). These additional effects will be demonstrated and discussed in the conference. 